SLAC-PUB-15852

LCLS-II-TN-13-01

December 2013

\title{
What limits the gap in a flat dechirper for an X-ray FEL?
}

\author{
K.L.F. Bane and G. Stupakov
}




\section{INTRODUCTION}

In a linac-based X-ray free electron laser (FEL) there is often a need for energy chirp control of the beam before it enters the undulator. In Ref. [1] a corrugated, metallic beam pipe in round geometry is suggested and analyzed as a device that can be used to passively "dechirp" the beam. Compared to round geometry, flat geometry-having the beam pass between two flat, corrugated plates - has the advantage of allowing the dechirper strength to be adjustable by changing the separation of the plates. And, indeed, such a device was recently tested in a proof-of-principle experiment at the PAL injector test facility in Pohang, Republic of Korea [2], and another at BNL's ATF [3]. One disadvantage of flat geometry is that, in addition to the usual dipole wakefield that is excited when the beam passes through the structure off axis, there is also a quadrupole wake excited, even when structure and beam are perfectly aligned.

For analyzing the wake effects for a bunch moving near the axis of a flat dechirper, one needs to consider the longitudinal, dipole, and quadrupole wakefields (we assume that the transverse extent of the bunch is small compared to the structure gap). We denote the longitudinal, (vertical or $y$ ) dipole, and (vertical or $y$ ) quad point charge wakes with the symbols $W_{z}(s), W_{q}(s), W_{d}(s)$, respectively; here $s$ is the longitudinal distance between a leading (or driving) particle and a test particle. The total transverse wakes at the test particle, in both vertical $(y)$ and horizontal $(x)$ directions, are given by

$$
W_{y}(s)=y_{l} W_{d}(s)+y W_{q}(s), W_{x}(s)=\left(x_{l}-x\right) W_{q}(s)
$$

with $y_{l}(y)$ the transverse position, with respect to the axis, of the leading (test) particle. We here assume that the dechirper is composed of two plates, that are oriented horizontally. 
In this note we provide analytical estimates of the three functions $W_{z}(s)$, $W_{q}(s), W_{d}(s)$, and their effects on energy chirp and projected emittance. We, in addition, apply these formulas to a set of beam and machine parameters applicable to the Linac Coherent Light Source (LCLS) [4]. Finally, we try to answer the question: what is the minimum useful dechirper gap given the strength of the transverse wakefields? The motivation of this work is a proposal by RadiaBeam Technologies to build such a device for use in the LCLS. A study that focuses specifically on that project, while considering also applications to the LCLS upgrade project-LCLS-II [5] — is given in Ref. [6].

Parameters used in the calculations of this report are given in Table I. Finally, note that a detailed study of the longitudinal wake effect in a corrugated structure in flat geometry can be found in Ref. [7].

TABLE I. Typical LCLS parameters, used in the example calculations.

\begin{tabular}{||l|r|r||}
\hline Parameter name & Value & Unit \\
\hline \hline Beam energy, $E$ & 6.6 & $\mathrm{GeV}$ \\
Beam charge, $Q$ & 250 & $\mathrm{pC}$ \\
Bunch length (rms), $\sigma_{z}$ & 10 & $\mu \mathrm{m}$ \\
Focusing function, $\beta_{y}$ & 10 & $\mathrm{~m}$ \\
Dechirper length, $L$ & 1 & $\mathrm{~m}$ \\
\hline \hline
\end{tabular}

\section{LONGITUDINAL WAKE}

For a sufficiently short bunch the wake of a dechirper can be assumed constant, and after passing through a structure of length $L$ the energy difference of the tail compared to the head (assuming flat geometry) is

$$
\Delta E=-W_{z}\left(0^{+}\right) Q L=-\left(\frac{\pi^{2}}{16}\right)\left(\frac{Z_{0} c}{\pi a^{2}}\right) Q L,
$$


with $W_{z}\left(0^{+}\right)$the longitudinal wake just behind the driving particle, $Q$ the bunch charge, $Z_{0}=377 \Omega, c$ the speed of light, and $a$ the half-aperture of the dechirper.

In Fig. 1 we plot the energy difference between the tail and the head of the beam as function of $a$, assuming charge $Q=250 \mathrm{pC}$ and structure length $L=1 \mathrm{~m}$. Note that after the structure, at half-gap $a=1 \mathrm{~mm}$, the tail of the bunch has lost $-\Delta E=5.4 \mathrm{MeV}$ compared to the head.

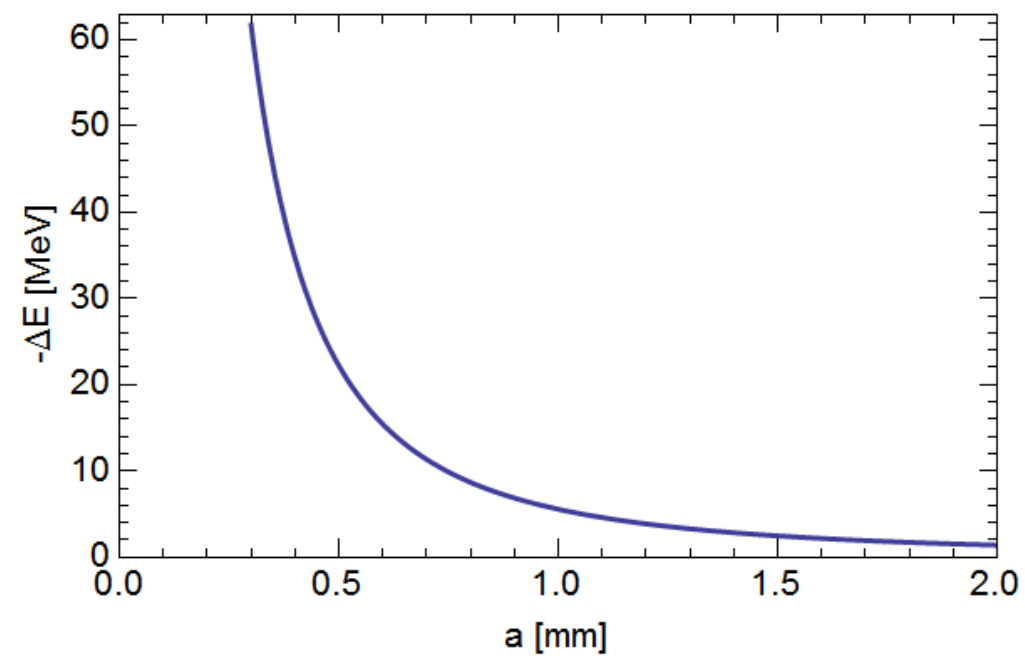

FIG. 1. Energy difference at tail of a short bunch compared to the head due to the wakefield of a flat dechirper vs. half-aperture $a$. Here charge $Q=250 \mathrm{pC}$ and structure length $L=1 \mathrm{~m}$.

\section{QUADRUPOLE WAKE}

Assume that we have a bunch with a symmetric transverse charge distribution. If a test particle within the bunch, at longitudinal position $s$, is offset 
from the dechirper axis by $y$ then it experiences a kick given by

$$
\Delta y^{\prime}(y, s)=y \alpha_{q}(s),
$$

with $\alpha_{q}(s)$ the inverse (vertical) focal length due to the quad wakefield (the quad wake is defocusing in $y$ and focusing in $x$ if the two plates of the dechirper are oriented so that one is above the other). The function $\alpha_{q}(s)$ depends on the bunch shape. If the initial two dimensional transverse distribution function is given by $\rho\left(y, y^{\prime}\right)$, then

$$
\alpha_{q}(s)=\frac{Q L}{E} W_{\lambda q}(s),
$$

with $E$ the beam energy. The bunch quad wake is given by

$$
W_{\lambda q}(s)=\frac{Q L}{E} \int_{0}^{\infty} W_{q}\left(s^{\prime}\right) \lambda\left(s-s^{\prime}\right) d s^{\prime},
$$

with $W_{q}(s)$ the quad point charge wake, and $\lambda(s)$ the longitudinal bunch distribution.

The emittance of the beam can be written as

$$
\epsilon_{y}^{2}=\sigma_{y}^{2} \sigma_{y^{\prime}}^{2}-\sigma_{y y^{\prime}}^{2}
$$

with $\sigma_{y}, \sigma_{y^{\prime}}$, the second moments (the standard deviations) of the bunch distribution in transverse phase space, and $\sigma_{y y^{\prime}}$ the correlation moment. The transformation described by Eq. 3 converts initial to final coordinates, $\left(y, y^{\prime}\right) \rightarrow$ $\left(y, y^{\prime}+\alpha(s) y\right)$, and initial to final distributions $\rho\left(y, y^{\prime}\right) \rightarrow \rho\left(y, y^{\prime}-\alpha(s) y\right)$, since the transformation is area preserving. Then assuming the initial distribution $\rho\left(y, y^{\prime}\right)$ is bi-gaussian with no correlations, we find that after the dechirper

$$
\begin{gathered}
\sigma_{y^{\prime}}^{2}=\int \lambda(s) d s \int d y \int\left(y^{\prime}\right)^{2} \rho\left(y, y^{\prime}-\alpha_{q}(s) y\right) d y^{\prime}=\sigma_{y^{\prime} 0}^{2}+\sigma_{y 0}^{2}\left\langle\alpha_{q}^{2}\right\rangle, \\
\sigma_{y y^{\prime}}=\int \lambda(s) d s \int d y \int y y^{\prime} \rho\left(y, y^{\prime}-\alpha_{q}(s) y\right) d y^{\prime}=\sigma_{y 0}^{2}\left\langle\alpha_{q}\right\rangle,
\end{gathered}
$$


and $\sigma_{y}=\sigma_{y 0}$ remains unchanged (note that $\left\langle y^{\prime}\right\rangle$ is zero). In these equations subscript 0 means a nominal size, and \langle\rangle denotes averaging a function over a distribution. Combining these results and taking $\sigma_{y 0}^{2}=\beta_{y} \epsilon_{y 0}, \sigma_{y^{\prime} 0}^{2}=\epsilon_{y 0} / \beta_{y}$, with $\beta_{y}$ the focusing function at the dechirper, we find that

$$
\left(\frac{\epsilon_{y}}{\epsilon_{y 0}}\right)^{2}=1+\beta_{y}^{2}\left(\alpha_{q}\right)_{r m s}^{2}
$$

where $\left(\alpha_{q}\right)_{r m s}$ is the rms of the inverse focal length over the longitudinal distribution $\lambda$.

The transverse point charge wakes begin as a linear function of $s$. Let us assume the bunch is short enough for this approximation to be valid over the whole bunch. Then

$$
W_{q}(s)=\left(\frac{\pi^{4}}{32}\right)\left(\frac{Z_{0} c}{4 \pi}\right) \frac{s}{a^{4}} H(s),
$$

with $H(s)=1(0)$ for $s>0(s<0)$.

Let us next assume for simplicity that the bunch distribution is uniform, i.e. that $\lambda(s)=\ell^{-1}$ for $|s|<\frac{\ell}{2}$, and that it equals 0 for $|s|>\frac{\ell}{2}$. The quad point charge wake is of the form $W_{q}=A H(s) s$, and the bunch wake becomes

$$
W_{\lambda q}(s)=\frac{A \ell}{2}\left(\frac{s}{\ell}+\frac{1}{2}\right)^{2}
$$

The first and rms moments of this function are: Al times, respectively, $1 / 6$ and $1 / \sqrt{45}$. Combining all the factors (noting that $\ell=2 \sqrt{3} \sigma_{z}$ for a uniform distribution) we find the projected emittance growth due to the quad wake of the flat dechirper is

$$
\left(\frac{\epsilon_{y}}{\epsilon_{y 0}}\right)^{2}=1+\left[(0.125) Z_{0} c \frac{Q \beta_{y} \sigma_{z} L}{a^{4} E}\right]^{2} .
$$

In Fig. 2 we plot $\delta \epsilon_{y} \equiv\left(\epsilon / \epsilon_{0}\right)-1$ vs. $a$, for the LCLS parameters of Table I. We see that for these parameters, the half aperture of the dechirper 
needs to be kept to $a \gtrsim 0.6 \mathrm{~mm}$, if one wants $\delta \epsilon_{y}<0.08$. As $a$ becomes smaller, the emittance grows steeply. Moving to smaller $a$ by modifying other parameters is not easy. If one wanted to reduce $a$ by $e . g$. a factor of two to $0.3 \mathrm{~mm}$, because of the $a^{-4}$ dependence, one would also need to reduce the combination of other parameters in the numerator (in the brackets) of Eq. 12 by a factor of 16 ! However, note that, if one made a dechirping structure composed of two identical parts, with the second half rotated by 90 degrees with respect to the first half, and with $\beta_{x}=\beta_{y}$, then the quad wake effect will cancel (we will discuss this idea again later).

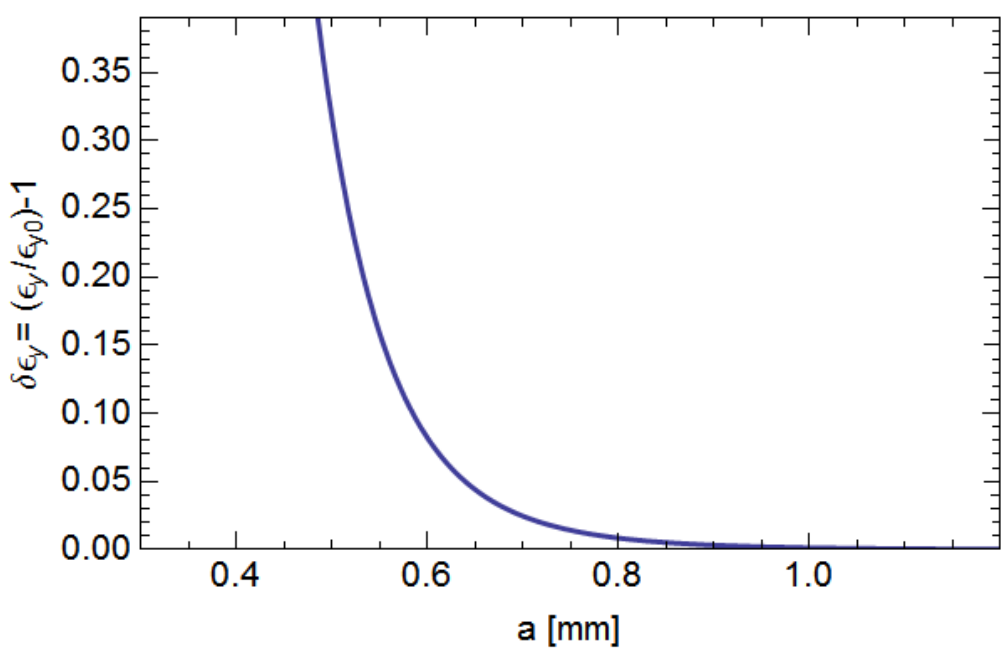

FIG. 2. Relative emittance growth due to the quad wake in a flat dechirper vs. half-aperture $a$. Here charge $Q=250 \mathrm{pC}$, energy $E=6.6 \mathrm{GeV}$, rms bunch length $\sigma_{z}=10 \mu \mathrm{m}$, and structure length $L=1 \mathrm{~m}$. The beam is on-axis; it has a longitudinal uniform distribution and, initially, an uncorrelated, bi-gaussian transverse distribution in $y y^{\prime}$ phase space.

From this analysis we can specify a minimum acceptable value of half aper- 
ture, $a_{m i n}$, as one that generates an allowable relative emittance growth (by which we mean $\left.\epsilon / \epsilon_{0}-1\right), \delta \epsilon_{t o l}$,

$$
a_{m i n}=\delta \epsilon_{t o l}^{-1 / 8}\left[(0.088) Z_{0} c \frac{Q \beta_{y} \sigma_{z} L}{E}\right]^{1 / 4} .
$$

Because of the small power dependencies on the parameters $\left(-\frac{1}{8}, \frac{1}{4}\right)$ we see that $a_{\text {min }}$ is rather insensitive to all parameters. For the LCLS parameters, assuming an allowable level of emittance growth $\delta \epsilon_{t o l}=10 \%$, we find that $a_{\min }=0.6 \mathrm{~mm}$.

\section{DIPOLE WAKE}

If the beam enters the structure vertically offset from the axis by $y=y_{0}$, it will excite both dipole and quadrupole wakefields. The kick on a test particle in a bunch (assuming the $y$ distribution is symmetric) becomes

$$
\Delta y^{\prime}(y, s)=y \alpha_{q}(s)+y_{0} \alpha_{d}(s)
$$

with the inverse dipole focal length $\alpha_{d}(s)$ defined analogously to $\alpha_{q}(s)$ (Eq. 4), using the dipole wake functions $W_{d}(s)$ and $W_{\lambda d}(s)$. Let us here assume we are interested in the effect of an injection jitter error in $y$. By a jitter error we mean an error that cannot be corrected. Consequently, the emittance should be calculated with respect to the structure origin; i.e.

$$
\bar{\epsilon}_{y}^{2}=\left\langle y^{2}\right\rangle\left\langle\left(y^{\prime}\right)^{2}\right\rangle
$$

The calculation is similar to the case of the quad wake effect. In this case, however, the initial $y$ distribution is Gaussian with rms size $\sigma_{y 0}$ and offset $y_{0}$; consequently, $\left\langle y^{2}\right\rangle=\sigma_{y 0}^{2}+y_{0}^{2}$. The second moment in $y^{\prime}$ is given by

$$
\begin{aligned}
\left\langle\left(y^{\prime}\right)^{2}\right\rangle & =\int \lambda(s) d s \int d y \int\left(y^{\prime}\right)^{2} \rho\left[y, y^{\prime}-\alpha_{q}(s) y-\alpha_{d}(s) y_{0}\right] d y^{\prime} \\
& =\sigma_{y^{\prime} 0}^{2}+y_{0}^{2}\left\langle\left(\alpha_{q}+\alpha_{d}\right)^{2}\right\rangle+\sigma_{y 0}^{2}\left\langle\alpha_{q}^{2}\right\rangle .
\end{aligned}
$$


We arrive at [to leading order in $\left.\left(y_{0} / \sigma_{y 0}\right)\right]$

$$
\left(\frac{\bar{\epsilon}_{y}}{\epsilon_{y 0}}\right)^{2}=1+\left[1+\beta_{y}^{2}\left\langle\left(\alpha_{q}+\alpha_{d}\right)^{2}\right\rangle\right]\left(\frac{y_{0}}{\sigma_{y 0}}\right)^{2}+\beta_{y}^{2}\left\langle\alpha_{q}^{2}\right\rangle .
$$

For a relative jitter amplitude of $\left(y_{0} / \sigma_{y 0}\right)$ this equation gives the relative emittance growth. Of the three terms in the above equation, the second gives a dipole-like contribution and the third a quad contribution to the emittance growth. We note that, in the dipole-like term, $\alpha_{q}$ and $\alpha_{d}$ contribute equally. In a linac-based, X-ray FEL an acceptable transverse jitter in the undulator (even without wakefield effects) is small compared to 1, e.g. the acceptable $\left(y_{0} / \sigma_{y 0}\right) \sim 0.2$. When $\left(y_{0} / \sigma_{y 0}\right) \ll 1$, we see from Eq. 17 that, in terms of effect on projected emittance, the quad wake term dominates the dipole wake term, which can thus be ignored.

If, as discussed above, we build a dechirper that comprises two identical halves, and rotate one half by 90 degrees with respect to the other, and at the same time set $\beta_{x}=\beta_{y}$ at the structure, then, in principle, we can cancel the quad wake effect. In such a case we are still left with the dipole wake effect, which will be half as strong as before, but now in both $x$ and $y$.

For the dipole wake effect in $y$, let us denote as injection jitter amplification factor the term

$$
\xi=\left[1+\beta_{y}^{2}\left\langle\left(\alpha_{q}+\alpha_{d}\right)^{2}\right\rangle\right]^{1 / 2}
$$

For small argument, the amplification factor can be written in the familiar form, $\xi \approx 1+\frac{1}{2} \Upsilon^{2}[8]$, with the strength factor here given by $\Upsilon=\beta_{y}\left\langle\alpha_{q}+\alpha_{d}\right\rangle$. As in the previous section we assume that the bunch is short enough that the initial linear rise of the dipole wake is valid. In this regime the dipole wake equals in amplitude the quad wake, i.e. $\alpha_{d}(s)=\alpha_{q}(s)$. The equations for the quad wake, Eqs. 4, 10, 11, are the same as the corresponding ones for the 
dipole wake. The injection jitter amplification factor $\xi$

$$
\xi=\left(1+\left[(0.375) Z_{0} c \frac{Q \beta_{y} \sigma_{z} L}{a^{4} E}\right]^{2}\right)^{1 / 2} .
$$

The amplification factor is plotted in Fig. 3 as function of half aperture $a$ for the example LCLS parameters used above. We see that one needs to keep the half aperture of the dechirper jaws to $a \gtrsim 0.7 \mathrm{~mm}$ for $\xi \lesssim 1.2$.

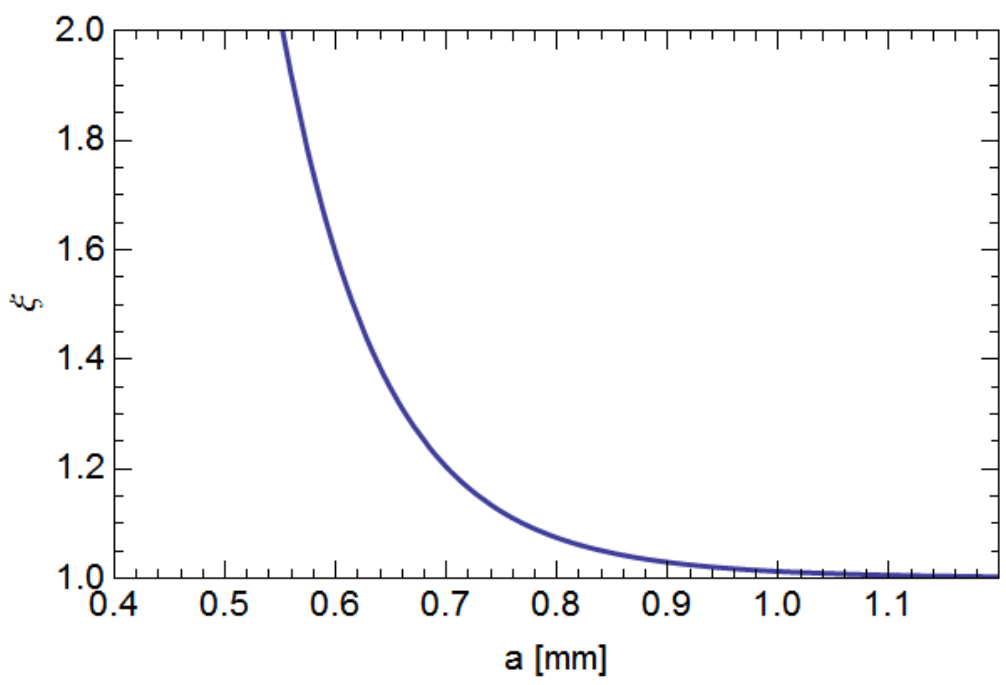

FIG. 3. Jitter amplification factor $\xi$ due to the dipole wake in a flat dechirper $v s$. half-aperture $a$. Here charge $Q=250 \mathrm{pC}$, energy $E=6.6 \mathrm{GeV}$, rms bunch length $\sigma_{z}=10 \mu \mathrm{m}$, and structure length $L=1 \mathrm{~m}$. The beam has a longitudinal uniform distribution, and an uncorrelated, bigaussian transverse distribution in $y y^{\prime}$ phase space.

\section{QUAD WAKE COMPENSATED DECHIRPER}

We have shown that if we use a single dechirper, the quad wake effect will dominate the emittance growth, assuming that the injection jitter amplitude 
$\left(y_{0} / \sigma_{y 0}\right) \ll 1$. We have also shown that if the dechirper is composed of two identical parts, with one rotated by 90 degrees with respect to the other, and if further $\beta_{x}=\beta_{y}$, then the quad wake effect cancels and we are left with the dipole effect alone. Let us analyze this scenario further.

Let us consider a simple $L=2 \mathrm{~m}$ structure in the LCLS with the example parameters used above, and let us consider the case of half gap $a=0.75 \mathrm{~mm}$. The emittance growth due to the quad wake is $\delta \epsilon_{y}=5.5 \%$ and the jitter amplification factor $\xi=1.43$. For the compensated dechirper composed of two identical parts rotated $90^{\circ}$ with respect to each other, the quad wake will be zero. However, if the gap of the two parts differs by $\Delta a$, then the residual inverse focal length $\left(\alpha_{q}\right)_{r m s}$ will be smaller by the factor $(2 \Delta a / a)$. For the same emittance effect as the original $2 \mathrm{~m}$ structure, one can reduce the aperture of the compensated structure by the factor $(2 \Delta a / a)^{1 / 4}$ [because of the $L / a^{4}$ scaling]. For an accuracy e.g. $\Delta a=30 \mu \mathrm{m}$ with $a=0.75 \mathrm{~mm}$, this factor is 0.53 , and the aperture of the compensated structure can be reduced to $a=0.53 * 0.75 \mathrm{~mm}=0.40 \mathrm{~mm}$. This would suggest that the strength of the longitudinal wake can be increased by the factor $(0.75 / 0.40)^{2}=3.5$.

However, if this is done, the injection jitter amplification factor, now equal in both planes, will become large. With the original structure it was $\xi=1.43$ (in $y$ ), now it becomes $\xi=6.4$ (in both $x$ and $y$ ). We can keep both the quad emittance growth small and the jitter amplification factor down to, say, $\xi=2$; this implies that $a=0.55 \mathrm{~mm}$, and we still gain a factor $(.75 / .55)^{2}=1.9$ in longitudinal effect over the simple $2 \mathrm{~m}$ structure. One needs to decide whether this factor of 2 in longitudinal effect is worth the added complication in design and operation of a partially compensated structure. 


\section{CONCLUSION}

Consider a short bunch of charge $Q=250 \mathrm{pC}$ and rms length $\sigma_{z}=10 \mu \mathrm{m}$, and a beta function $\beta_{y}=10 \mathrm{~m}$. For a simple, $L=2 \mathrm{~m}$ structure, with half-aperture $a=0.75 \mathrm{~mm}$, the head-to-tail energy difference produced by the dechirper wake is $\Delta E=-19.8 \mathrm{MeV}$. The emittance effect of the quad wake is negligible, and the dipole wake amplification factor (in $y$ ) $\xi=1.43$. For a more complicated device, composed of two identical $1 \mathrm{~m}$ structure, but with one rotated by 90 degrees, with $a=0.55 \mathrm{~mm}$ and assuming $\beta_{x}=\beta_{y}$, $\Delta E=36.8 \mathrm{MeV}$. Assuming the half-gap in the two halves is the same to a tolerance of $\Delta a=30 \mu \mathrm{m}$, then again the emittance effect of the quad wake is negligible, and the dipole wake amplification factor $\xi=2$ (in both $x$ and $y$ ).

Two important assumptions in all calculations are: (1) that the bunch is sufficiently short so that the leading order of the point charge wakes applies, and (2) that the leading order of the short-range wakes agrees with their analytical value. When the actual parameters of a candidate dechirper are being decided, a field matching program [7] can be used to obtain more accurate results than given here.

\section{ACKNOWLEGEMENTS}

We also thank Z. Huang for helpful discussions on the subject of this note. Work supported by Department of Energy contract DE-AC02-76SF00515.

[1] K. Bane and G. Stupakov, Nucl. Inst. Meth. A690 (2012) 106.

[2] P. Emma et al, "Experimental demonstration of energy-chirp control in relativistic electron bunches using a corrugated pipe," submitted to PRL, October 
2013.

[3] M. Harrison et al, Proc. of NaPAC13, Pasadena, CA, contribution MOPHO25, September 2013.

[4] P. Emma et al, Nat. Photonics 4, 641 (2010).

[5] "Linac Coherent Light Source II Conceptual Design Report," report in preparation, December 2013.

[6] K. Bane et al, "A Possible Dechirper Device for the LCLS," SLAC-PUB-15853, December 2013.

[7] K. Bane and G. Stupakov, Phys. Rev. ST Accel. Beams 6 (2003) 023301.

[8] A. Chao, Physics of collective beam instabilities in high energy accelerators, (John Wiley \& Sons, 1993) p. 150. 